\title{
PROJETO SEXUALIDADE RESPONSÁVEL: AÇÕES DESENVOLVIDAS POR ACADÊMICOS DE ENFERMAGEM
}

\section{PROJECT "RESPONSIBLE SEXUALITY": THE ACTIONS DEVELOPED BY NURSING STUDENTS}

\author{
Débora Leão Alves* \\ ORCID: https://orcid.org/0000-0003-2029-386X \\ Jeyce Kelly da Silva Messias** \\ ORCID: https://orcid.org/0000-0003-0002-9535 \\ Gabriela Larissa Vieira Pinto*** \\ ORCID: https://orcid.org/0000-0003-0790-2130 \\ Nayane de Sousa Silva Santos**** \\ ORCID: http://orcid.org/0000-0002-1668-5505 \\ Mirian Cristina dos Santos Almeida***** \\ ORCID: https://orcid.org/0000-0002-9178-1345 \\ Ulisses Vilela Hipólito****** \\ ORCID: https://orcid.org/0000-0003-0353-6479
}

\section{Resumo}

Objetivou-se descrever as atividades voltadas ao enfrentamento das Infecções Sexualmente Transmissíveis (IST) desenvolvidas pelo Projeto de Extensão Sexualidade Responsável. O projeto é caracterizado como estudo descritivo, do tipo relato de experiência. Após treinamento teórico-prático, os extensionistas realizaram atividades de educação em saúde sobre IST e testagens rápidas para hepatites B e C, sífilis e HIV, além de distribuírem, ao todo, 15.536 preservativos e 8.000 géis lubrificantes. As ações aconteceram entre outubro de 2018 a março de 2020, tendo como população-alvo alunos, funcionários de instituições de ensino e comunidade em geral. Receberam treinamento 57 extensionistas. Foram realizadas 13 ações, em sua maioria em instituições educacionais. Dos 573 indivíduos testados em ações do projeto, detectaram-se 16 resultados reagentes para sífilis e 2 de HIV. Ao todo, foram realizados 2.294 testes rápidos. Conclui-se que a extensão universitária gerou impactos positivos na formação dos alunos, ao mesmo tempo que contribuiu com o enfrentamento das IST.

Palavras-chave: Doenças Sexualmente Transmissíveis; Extensão Comunitária; Enfermagem.

Data recebimento: $13 / 09 / 2021$

Data de aceite: $23 / 12 / 2021$

\begin{abstract}
This study aims to describe the activities developed by the outreach project "Responsible Sexuality" at combating sexually transmitted diseases (STIs). It is a descriptive study developed through experience report. After theoretical and practical training, it was conducted health education activities on STIs, distributed condoms and lubricating gels and performed rapid tests for hepatitis B and C, syphilis and HIV. The actions were carried out from October 2018 to March 2020 and targeted at students and staff from educational institutions and community in general. 57 people were training; 13 actions were carried out in educational institutions; 15.536 condoms and 8.000 lubricating gels were distributed; 573 individuals were tested; 16 reactive cases of syphilis and 2 HIV were detected; 2.294 rapid tests were performed. It is possible to conclude that the outreach actions had positive impacts on the formation of students and helped them to confront STIs.
\end{abstract}

Keywords: Sexually Transmitted Diseases; Community-Institutional Relations; Nursing. 


\section{Introdução}

No Brasil, as Infecções Sexualmente Transmissíveis (IST) tornaram-se um grande problema de saúde pública devido ao seu crescimento nos últimos anos. Essas infecções têm múltiplas etiologias e apresentações clínicas, e impactam na qualidade de vida das pessoas, nas relações pessoais, familiares e sociais (BRASIL, 2015a). Elas são causadas por protozoários, bactérias e vírus, entre outros microrganismos e são transmitidas principalmente por via vaginal, anal e oral sem o uso de preservativos, através de um indivíduo que esteja infectado para outros (BRASIL, 2019a). Entre as IST com maior impacto sobre a população brasileira estão a Sífilis, HIV, Hepatites B e C.

As referidas IST são muitas vezes silenciosas, podendo ficar meses e até anos sem apresentarem sinais e sintomas. O HIV é um vírus causador da Síndrome da Imunodeficiência Adquirida (AIDS), que ataca o sistema imunológico, o qual é responsável por proteger o organismo. De 2007 até junho de 2019, foram notificados no Sistema de Informação de Agravos de Notificação (SINAN) 300.496 casos de infecção pelo HIV no Brasil (BRASIL, 2019b), mostrando que ainda existem pontos a serem melhorados nas políticas de prevenção das IST.

Já a sífilis é uma doença infecciosa causada pela bactéria Treponema Pallidum e se desenvolve em quatro estágios: primário, secundário, latente e terciário. O primeiro estágio geralmente envolve uma ferida indolor na genitália, no colo uterino ou em outros locais da pele, denominada "cancro duro". Por ser uma ferida indolor, dificilmente diagnosticada nesta etapa. O segundo estágio se inicia com a disseminação do Treponema Pallidum pelo organismo, o que ocorre entre 6 semanas e 6 meses após a cicatrização do cancro e pode durar de 4 a 12 semanas, sendo caracterizada principalmente pela presença de pápulas palmo plantares, placas e condilomas planos que desaparecem com ou sem tratamento. Logo após, se inicia o estágio de latência, dividido em até 2 anos de infecção latente recente e mais de 2 anos de infecção latente tardia, permanecendo de 1 até 40 anos, quando se inicia o terceiro estágio, com retorno da presença de manifestações clínicas, através de lesões mais graves em diversos órgãos e tecidos, podendo levar à morte (BRASIL, 2019c). No Brasil, em 2018, foram notificados 158.051 casos de sífilis adquirida (BRASIL, 2019d).

Uma das maiores preocupações relacionadas à sífilis é a transmissão vertical ou sífilis congênita, pois, caso ela ocorra, o recém-nascido poderá nascer com malformações e há até mesmo o risco de morte perinatal. A sífilis congênita é diferenciada em precoce, quando as manifestações clínicas surgem até o segundo ano de vida, e tardia, quando estas surgem após os 2 anos de idade. Na sífilis congênita precoce, as anormalidades radiológicas são as mais comuns, acometendo cerca de 70 a 100\% dos casos, podendo ainda apresentar reações sistêmicas, muco cutâneas, hematológicas, neurológicas e outras. Já na sífilis congênita tardia, são comuns manifestações oftalmológicas, auditivas, orofaríngeas, cutâneas, alterações faciais e esqueléticas, e prejuízos ao sistema nervoso central (BRASIL, 2019d). Todos esses problemas acarretam sérias consequências ao desenvolvimento infantil e geram gastos à saúde pública. 
As hepatites virais são doenças que podem ser causadas por cinco tipos diferentes de vírus, todos com propensão de infecção primária do tecido hepático. Em sua maioria, as hepatites virais agudas não apresentam sintomas, mas, quando apresentam, são comuns a fadiga, mal-estar, náuseas, dor abdominal, anorexia e icterícia. As hepatites crônicas, normalmente, progridem de forma assintomática (BRASIL, 2009).

Pode-se diferenciar as hepatites virais de acordo com suas formas de transmissão. As hepatites A e E são transmitidas por via fecal-oral, ou seja, através de água, alimentos, mãos e utensílios contaminados com fezes contendo os vírus que são levados à boca. Já a $\mathrm{B}, \mathrm{C}$ e $\mathrm{D}$ são transmitidas pelo sangue e seus hemoderivados, através de materiais contaminados, relação sexual desprotegida, órgãos transplantados e pela transmissão vertical. No entanto, o vírus da hepatite D (HDV, do inglês hepatitis D virus) depende do vírus da hepatite B (HBV, do inglês hepatitis $B$ virus) para se multiplicar, necessitando, assim, de uma coinfecção ou infecção prévia por HBV (BRASIL, 2014).

Ressalta-se que, dentre as hepatites virais, os testes rápidos são feitos somente para as Hepatites B e C, uma vez que estas têm transmissão por via sexual, portanto, são consideradas ITS, e que o vírus da hepatite $\mathrm{D}$ deve ser investigado somente em indivíduos com resultados reagentes para HBV e que residem ou estiveram em áreas endêmicas para hepatite D (BRASIL, 2018a).

No diagnóstico inicial dessas infecções, preconiza-se o uso de Testes Rápidos (TR), por serem testes práticos e de fácil execução, com a leitura do resultado disponível em até 30 minutos, o que viabiliza o início do tratamento imediatamente após o diagnóstico (BRASIL, 2020). É importante salientar que somente profissionais treinados estão aptos a realizar os TR.

Para tanto, é necessário seguir um Procedimento Operacional Padrão (POP), contendo as seguintes etapas: identificação e cadastramento do usuário; tipos de amostra; etapas de realização do teste; interpretação do resultado; e emissão do laudo (BRASIL, 2010). Ademais, as etapas de realização dos TR incluem o pré-teste e o pós-teste, onde é esclarecido sobre quais testes serão realizados, assim como a qualidade deles, o significado de cada possível resultado, tratamentos disponíveis e, ainda, sobre vulnerabilidade e janela imunológica (BRASIL, 2018b).

A praticidade dos TR possibilita sua utilização em diversas situações, como atividades de rastreamento intra e extramuros, após contato sexual desprotegido (consentido ou não), acidentes com materiais perfuro cortantes, na admissão de gestantes que não realizaram o prénatal, dentre outras situações. Dessa forma, os TR propiciam maior cobertura populacional, principalmente às populações isoladas, visto que não há a necessidade de estrutura laboratorial para a sua execução (BARTH, 2018).

As facilidades de implementação dos TR corroboram o diagnóstico precoce das IST em questão. Ao realizar o diagnóstico precoce da sífilis, por exemplo, há a possibilidade de prevenção da transmissão vertical e tratamento oportuno (BAGATINI, 2016). Para a hepatite 
B e C, é possível, assim, ofertar um tratamento adequado e prevenir complicações, como o câncer hepático e a cirrose avançada (BRASIL, 2018a). O tratamento precoce do HIV é capaz de preservar e reconstruir o sistema imunitário, diminuindo a mortalidade e probabilidade de transmissão (LORETO, 2012).

Tendo em vista todos os pontos citados anteriormente, o Curso de Enfermagem de uma universidade do estado do Tocantins criou um projeto de extensão intitulado Sexualidade Responsável, objeto deste estudo, que tem por objetivo orientar a população em relação aos meios de prevenção de doenças sexualmente transmissíveis, com foco principal no HIV, sífilis e hepatites $\mathrm{B}$ e $\mathrm{C}$, realizar triagem sorológica e diagnóstico dessas doenças através da realização de testes rápidos. Esse projeto mostra sua importância devido ao aumento do número de casos de IST em nossa população e pelo fato de o diagnóstico e tratamento correto das referidas infecções poder interromper o ciclo de transmissão e até mesmo curar algumas delas, prevenindo agravos e mortes.

Desta forma, o presente trabalho tem por objetivo descrever as atividades voltadas ao enfrentamento das IST desenvolvidas pelo Projeto de Extensão Sexualidade Responsável.

\section{Método}

Trata-se de um estudo descritivo, do tipo relato de experiência, realizado por estudantes participantes do projeto de extensão intitulado Sexualidade Responsável, do Curso de Enfermagem da Universidade Federal do Tocantins (UFT). Após um treinamento teóricoprático sobre IST, oferecido na disciplina Cuidados Especiais nos Ciclos da Vida, em parceria com a Secretaria Municipal de Saúde de Palmas - TO, os extensionistas desenvolveram grupos de educação em saúde sobre IST, distribuição de preservativos, géis lubrificantes, e realizaram testagens rápidas para sífilis, HIV e hepatites B e C, incluindo as etapas de educação em saúde, com aconselhamento no pré e pós-teste. As ações ocorreram no período de outubro de 2018 a março de 2020, no município de Palmas - TO, e uma ação em Porto Nacional - TO.

As atividades foram realizadas pelos acadêmicos de Enfermagem que já haviam passado, ou ainda estavam passando, pela disciplina denominada "Cuidados Especiais nos Ciclos da Vida", sendo um dos critérios de aprovação a participação nas ações de extensão durante o período em que o aluno estava matriculado na disciplina, mas o ingresso no projeto de extensão e contribuição com as ações após a finalização dela ficava a critério do aluno. Esses alunos eram previamente capacitados por meio de cursos teóricos gratuitos disponibilizados no TELELAB pelo Ministério da Saúde e com parte prática realizada em parceria com a Secretaria Municipal de Saúde de Palmas - TO, que aconteceram no próprio laboratório da universidade com profissionais da Secretaria de Saúde, colaboradores do projeto. Durante as atividades, eram supervisionados diretamente por professores participantes do 
projeto de extensão. Objetivou-se, com as ações realizadas, facilitar o acesso à informação, às medidas de prevenção sobre IST e aos testes rápidos para Sífilis, HIV e Hepatites $\mathrm{B}$ e C para alunos e funcionários de instituições de ensino e para comunidade em geral.

Nas ações feitas em instituições de ensino, contatou-se previamente os gestores para apresentação do projeto, agendamento de dias e horários compatíveis com os de maior fluxo de pessoas, disponibilização de espaço físico adequado para realização da testagem, orientações pré-teste e entrega dos resultados. Provisionaram-se os testes e insumos com Secretaria Municipal de Saúde ou com a Secretaria Estadual de Saúde, parceiras do Projeto "Sexualidade Responsável". Nas demais ações, os extensionistas foram convidados por organizadores de outros eventos a estarem presentes para fazer os testes rápidos.

Para a distribuição de preservativos e géis lubrificantes em duas instituições de ensino superior, realizou-se previamente o levantamento dos banheiros existentes e foram selecionados os dos blocos mais frequentados, onde fixaram-se caixas que eram abastecidas com preservativos e géis lubrificantes semanalmente pelos alunos extensionistas. A distribuição também ocorreu durante as ações de testagem rápida e de educação em saúde.

Durante as ações de testagem rápida, foram entregues panfletos informativos para as pessoas que estavam no local ou próximo a ele, divulgando a realização dos testes rápidos e sua importância. Após preencherem uma ficha de atendimento com dados pessoais, os participantes foram encaminhados à fase de pré-teste, que ocorreu em grupos de, em média, 5 pessoas; nessa fase, informava-se, por meio de uma conversa informal, como eram feitos os testes em si, quais as condutas em caso de testes reagentes, quais as IST testadas, seus modos de transmissão, janela imunológica e métodos de prevenção, dando oportunidade aos participantes de tirarem dúvidas no grupo ou de forma particular. O pós-teste aconteceu individualmente, quando foram entregues os resultados, reforçando as orientações e esclarecendo dúvidas. Todos os participantes que tiveram alguma IST detectada foram encaminhados para tratamento nas Unidades de Saúde, com agendamento.

Os dados apresentados foram coletados nos registros de Atividades do Projeto Sexualidade Responsável, resguardando a identidade dos indivíduos, o sigilo dos resultados dos exames, seguindo os preceitos éticos da Resolução n 510/2016 do Conselho Nacional de Saúde.

Os dados apresentados foram coletados nos registros de atividades do Projeto Sexualidade Responsável, digitados no Microsoft Excel 2016 e analisados por estatística descritiva simples, onde as informações encontram-se agregadas sem possibilidade de identificação dos participantes do projeto, resguardando a identidade dos indivíduos, o sigilo dos resultados dos exames, respeitando os preceitos éticos da Resolução n ${ }^{\circ}$ 510/2016 do Conselho Nacional de Saúde. 


\section{Resultados}

O Projeto de Extensão Sexualidade Responsável conta atualmente com 57 extensionistas, que receberam treinamento teórico-prático sobre IST e para a realização de testes rápidos. Os treinamentos aconteceram nos laboratórios da Universidade Federal do Tocantins, Campus Palmas. Ao todo, foram distribuídos 15.536 preservativos masculinos e femininos e 8.000 géis lubrificantes durante as ações e nos dispensadores nos banheiros de duas univer-sidades públicas.

Foram realizadas 13 ações no período de outubro de 2018 a março de 2020, sendo 3 em Universidade Federal, 2 em Universidade Estadual, 2 em Instituição pública de ensino tecnológico e superior, 1 na Universidade da Maturidade, 1 em Centro de Saúde Comunitária, uma em Estação de ônibus Apinajé, 1 no Terminal Rodoviário de Palmas, 1 Parque dos Povos Indígenas, e 1 em um Centro Comunitário na cidade de Porto Nacional-TO. Nessas 13 ações, 876 indivíduos realizaram testes rápidos para HIV, Hepatites B e C e Sífilis. Dessas, 3 ações ocorreram em parceria com outras instituições, ficando o cadastro dos usuários sob a responsabilidade delas. Assim, os dados apresentados a seguir são referentes às 10 ações realizadas sob responsabilidade exclusiva do Projeto Sexualidade Responsável, onde constatouse que foram testadas um total de 573 pessoas, sendo $334(58,3 \%)$ do sexo feminino e 239 $(41,7 \%)$ do sexo masculino.

Com relação à idade da população testada, a média foi de 30,3 anos, sendo 7 anos a idade da pessoa mais jovem e 80 anos a da pessoa mais idosa a ser testada. A faixa etária mais testada foi a de jovens de 15 a 24 anos, correspondendo a 59\% (338) do total de participantes, como mostra a Tabela 1.

Tabela 1 - Número de pessoas testadas de acordo com a faixa etária. Palmas-TO, 2018-2020.

\begin{tabular}{lrr}
\hline Faixa etária & Pessoas testadas \\
\cline { 2 - 3 } & $\mathbf{N}^{\mathbf{0}}$ & $\%$ \\
\hline Até 14 anos & 3 & $1 \%$ \\
\hline De 15 a 24 anos & 338 & $59 \%$ \\
\hline De 25 a 34 anos & 121 & $21 \%$ \\
\hline De 35 a 44 anos & 51 & $9 \%$ \\
\hline DE 45 a 54 anos & 19 & $3 \%$ \\
\hline De 55 a 64 anos & 23 & $4 \%$ \\
\hline De 65 ou mais & 18 & $3 \%$ \\
\hline TOTAL & $\mathbf{5 7 3}$ & $\mathbf{1 0 0 \%}$ \\
\hline
\end{tabular}

Fonte: Elaborado pelos autores. 
Pode ser observado na Tabela 2 que foram realizadas 2.294 testagens, nas quais 18 $(0,76 \%)$ tiveram resultados reagentes. Dos 18 testes reagentes, $16(88,88 \%)$ foram para sífilis, $2(11,11 \%)$ para HIV. Não houve nenhum resultado reagente para as hepatites B e C. Vale ressaltar que uma das instituições testadas não obteve nenhum resultado reagente. Dentre os testes reagentes, a faixa etária com maior número de reagentes foi também a de 15 a 24 anos, correspondendo a $61 \%$ (11) do total, seguida pela idade de 55 a 64 anos, com 17\% (4) do total de reagentes, apesar dessa faixa etária corresponder a somente $4 \%$ (23) do total de participantes.

Tabela 2 - Número de testes rápidos realizados e resultados reagentes para HIV, sífilis, hepatites B e C. Palmas - TO, 2018-2020.

\begin{tabular}{lcc}
\hline Testes & $\mathbf{N}^{\mathbf{0}}$ de testes realizados & Resultados reagentes \\
\hline HIV & 608 & 2 \\
\hline Sífilis & 647 & 16 \\
\hline Hepatite B & 445 & 0 \\
\hline Hepatite C & 594 & 0 \\
\hline TOTAL & 2294 & $\mathbf{1 8}$ \\
\hline
\end{tabular}

Fonte: Elaborado pelos autores.

Ainda sobre os testes reagentes, nos casos de sífilis, $11(68,75 \%)$ eram do sexo feminino, com idades entre 21 e 59 anos, e $5(31,25 \%)$ do sexo masculino, com idades entre 18 e 61 anos. Ambos testados para HIV eram do sexo masculino, com idades de 22 e 61 anos, e o paciente de 61 anos também foi detectado com sífilis, sendo este o único caso de coinfecção.

\section{Discussão}

A capacitação de novos atores para a realização de testes rápidos e acolhimento é uma importante estratégia para descentralização do diagnóstico e tratamento das IST (GOMES; GALINDO, 2017). Tal movimento pode contribuir para o aumento do número de pessoas alcançadas e acelerar o tempo que se levaria para a detecção de casos, evitando a disseminação das infecções e agravos. O Projeto de Extensão Sexualidade Responsável corrobora o 
fortalecimento da descentralização ao promover ações fora dos locais de serviços de saúde e, ao treinar os alunos ainda durante a sua formação acadêmica, expande-se, assim, o quantitativo de mão de obra qualificada, capacitada com habilidades técnicas e capaz de lidar com os aspectos emocionais dos usuários.

Tal como abordado no estudo de Silva et al. (2020), a extensão universitária tem um importante papel na criação de meios que favorecem a troca do conhecimento com a comunidade, viabilizando a construção de novos conhecimentos, possibilitando o descobrir e redescobrir de soluções e, por fim, reforçando o papel da universidade com a sociedade, a formação social dos alunos e a produção de conhecimentos.

Nesse projeto de extensão, além do treinamento para a realização de testes rápidos, um importante fator pensado e discutido anterior à implantação do projeto foi a localização dos dispensadores de preservativos e géis lubrificantes ou a forma de se dar acesso a eles, sendo a estratégia utilizada de fundamental importância para que realmente sejam adquiridos. Em pesquisa feita por Cunha (2015) e outra por Russo e Arreguy (2015), a partir de projetos relacionados à sexualidade, os participantes ressaltaram a importância de melhor acesso ao preservativo, por meio de estratégias que possibilitem menor constrangimento, e sugeriram locais como banheiros, biblioteca, corredores e salas de atendimento.

Quanto ao comparativo entre os números de homens e de mulheres participantes das ações do projeto, os achados do presente estudo reforçam outras literaturas científicas que mostram a menor utilização dos serviços de saúde pela população masculina. Tal fato pode ser explicado através do contexto difundido pelo modelo machista da sociedade, onde o homem é visto como um ser frágil ao buscar atendimento de saúde, indo contra a virilidade que se espera deles (SOUSA; SOUSA, 2017). Em contrapartida, estudos apontam para a maior adesão das mulheres às ações em saúde, principalmente aquelas que residem em contextos urbanos, brancas, com renda elevada e maior escolaridade (MARTINS; MODENA, 2017).

São inúmeros os fatores que reforçam a invisibilidade masculina no contexto de busca à saúde. Historicamente, os serviços de saúde têm maior foco no atendimento das demandas de mulheres, crianças e idosos, e menos na demanda do público masculino, muitas vezes até a desconhecendo (MARTINS; MODENA, 2017). Apesar de existirem políticas de saúde voltadas especificamente para os homens, em sua maioria seus problemas de saúde têm abordagens pontuais e descontinuadas, como campanhas de saúde e inclusão em grupos específicos, nos quais a preocupação com a saúde integral do próprio homem é relevada, e este é relacionado aos problemas que causam aos outros, como, por exemplo, transmissão de IST a parceiros, violência contra a mulher, entre outros (BOTTON; CÚNICO; STREY, 2017).

Essa menor adesão do sexo masculino aos serviços de saúde acaba gerando consequências para eles, como por exemplo um diagnóstico tardio e um pior prognóstico da doença. Para doenças, como por exemplo o HIV, o tempo entre a detecção da infecção e o início da 
terapia antirretroviral é um fator chave para evitar o avanço da doença, e desta forma facilitar a recuperação imunológica (AZEVEDO, 2017).

Assim como em outros estudos, as testagens abrangem em sua maioria o público mais jovem e feminino, mostrando sempre uma maior procura por parte deles. Com relação aos idosos, existe uma menor demanda por testagem principalmente devido à percepção ultrapassada de que nessa fase a vida sexual não é ativa. Para o HIV, tem sido demonstrado aumento significativo nas taxas entre homens e mulheres na faixa etária de 60 anos ou mais, nos últimos 10 anos, indicando a vulnerabilidade de idosos à sua ocorrência (ANDRADE et al., 2017).

A partir de busca na literatura sobre ações de testagem rápida realizadas no Brasil, acharam-se os seguintes dados em relação à prevalência de IST: em ação feita em Goiás com 481 pessoas, houve 2,49\% (12) de casos reagentes (NUNES et al., 2018); em uma ação feita em Minas Gerais com 757 pessoas, obtiveram-se 6\% (45) de testes reagentes, destes, 5,3\% eram reagentes para sífilis (BARBOSA et al., 2019). Já nas ações do Projeto Sexualidade Responsável relatadas no presente estudo, obteve-se uma prevalência de 3,14\% (18) reagentes para 573 participantes, sendo aproximadamente a mesma média de casos reagentes se comparada às outras ações, assim como maior prevalência para sífilis dentre as demais infecções.

A prevalência das IST será maior em estudos feitos com pessoas em situação de rua, privadas de liberdade, profissionais do sexo, entre outras. Em estudo feito por Barros et al. (2018) com 481 homens sem-teto, a prevalência de testes rápidos reagentes para sífilis foi de $10,2 \%$ (49); em estudo feito com 56 mulheres cumprindo regime semiaberto ou aberto, obtiveram-se 16,1\% (9) de casos reagentes em testes rápidos de sífilis (SILVA et al., 2018),

Apesar de menor prevalência de testes reagentes em relação a ações feitas com populações em situações de maior vulnerabilidade e de propensão a comportamentos de risco, as ações com universitários e em locais fora dos serviços de saúde se tornam necessárias, uma vez que o Ministério da Saúde estabeleceu o plano da tríplice meta 90-90-90, onde uma das metas inclui a ampliação do diagnóstico das pessoas que vivem com HIV (BRASIL, 2015b).

Verifica-se que não houve nenhum caso reagentes para hepatite $\mathrm{B}$ ou $\mathrm{C}$ durante as ações realizadas. Há, na literatura, condições associadas ao perfil das pessoas que adquirem essas hepatites, o que pode ter influenciado a não detecção de casos, visto que incluem populações mais específicas que podem não ter sido alcançadas, ou alcançadas minimamente. De acordo com revisão feita por Silva et al. (2019), alguns dos principais fatores associados ao desenvolvimento da hepatite B são: "a negligência dos profissionais de saúde com seringas e Equipamentos de Proteção Individual (EPI), indivíduos que compartilham seringas e canudos no uso de drogas, transplante de órgãos, principalmente do fígado", entre outros.

De acordo com dados do SINAN, a maior taxa de detecção de casos de hepatite está entre pessoas na faixa etária de 50 a 59 anos $(68,4 \%)$. Esta faixa etária correspondeu a apenas $4 \%$ dos testados durante as ações. O SINAN aponta, ainda, o estado do Tocantins como o de 
menor incidência de hepatite $\mathrm{C}$ no Brasil, e somente 5,7\% dos casos detectados pertencem à Região Norte. Quanto à provável fonte ou mecanismo de infecção, em sua maioria é desconhecido, seguido pelo uso de drogas $(12,6 \%)$ e a transfusão sanguínea $(10,8 \%)$ (BRASIL, 2019e).

Em consonância com a literatura, é possível identificar a vulnerabilidade às IST também em jovens universitários, sendo um dos motivos a falta de conhecimento sobre as IST, revelando, dessa forma, a importância da vulnerabilidade programática, ou seja, relacionada à necessidade de medidas que promovam a educação em saúde e impeçam a exposição aos agravos (BRUM; MOTTA; ZANATTA, 2019). Outro ponto contundente dos trabalhos sobre a temática destaca as desigualdades sociais e de gênero que refletem no cuidado com a saúde (FONTE et al., 2018). Corroborando a falta de conhecimento sobre IST, outro estudo mostrou que a maioria dos jovens não considera que o preservativo seja um método eficaz para a prevenção de doenças e da gravidez, os quais ainda mostram desconhecer as formas de contágio do HIV (FONTES et al., 2017). Esses dados demonstram uma grande falta do sistema de saúde no que diz respeito a ações de educação em saúde.

Com relação à quantidade de idosos com testes reagentes, haja vista a pouca quantidade de idosos testados durante as ações, um estudo sobre a vulnerabilidade desse grupo relacionou a alta prevalência de IST nessa faixa etária ao baixo uso de preservativos, e como uma das principais justificativas para isso a confiança em seus parceiros (FERREIRA et al., 2019). Outro fator que corrobora o aumento de IST nessa faixa etária é a vulnerabilidade individual, ou seja, o grau, qualidade e acesso a informações de saúde, o grau de escolaridade, os valores e crenças, que estão relacionados diretamente com comportamentos de risco do indivíduo no curso de sua vida, podendo deixá-lo mais suscetível a ter uma reinfecção ou um quadro infeccioso quando idoso (ANDRADE et al., 2017). Além disso, eles não se percebem nesse cenário de doença, por isso, não fazem uso adequado de preservativos (SANTOS, 2018). Logo, percebeu-se a necessidade de medidas de educação em saúde voltadas para a prevenção de IST que abordem as especificidades desse grupo.

Das IST detectadas, a sífilis foi a mais prevalente (88,8\% 16/18). Conforme o Boletim Epidemiológico de Sífilis (BRASIL, 2019d), nos últimos 10 anos, no Brasil, houve um aumento constante do número de novos casos de sífilis, que pode estar relacionado à ampliação do acesso ao diagnóstico através dos TR (BARTH; BECK, 2018; BRASIL, 2016), diminuição do uso de preservativos, falta de informações sobre a doença, tratamento inadequado (GUINSBURG et al., 2010), e às muitas dificuldades relacionadas à implantação do uso da penicilina nas unidades básicas de saúde (GRUMACH et al., 2007). Para o combate dessa epidemia, reforçase a importância da testagem de pessoas assintomáticas e o acompanhamento de todos os casos e de suas parcerias sexuais (BRASIL, 2020).

Neste estudo, revelou-se um maior número de mulheres testadas positivo para sífilis, achado que está em consonância com o Boletim Epidemiológico do Ministério da Saúde de 2019 , onde consta que 59,4\%, de um total de 853.937 casos notificados, entre 2010 e 2018, 
ocorreram em mulheres (BRASIL, 2019). Quanto ao perfil dessas mulheres, estudo realizado no interior paulista notou que a maioria mantinha relações apenas com homens, no entanto, não foi revelada multiplicidade de parceiros (ANDRADE et al., 2019). Em contrapartida, outro estudo feito com população em situação de rua, em São Paulo, apesar de as mulheres realizarem com mais frequência testes rápidos, o maior número de resultados positivos revelou-se entre os homens (PINTO et al., 2014).

No que tange à coinfecção por sífilis ou outras infecções, os estudos demonstram ser comum em pacientes infectados pelo HIV, caracterizando, dessa forma, um perfil epidemiológico. A coinfecção está diretamente ligada à depressão da imunidade desses pacientes, onde o déficit do sistema imunológico dá espaço às doenças oportunistas. Além disso, a maioria dos coinfectados é do sexo masculino, reafirmando o que foi evidenciado neste estudo. Esse achado pode ser justificado por comportamentos de risco do gênero (OLIVEIRA et al., 2019; ARAÚJO et al., 2017). No entanto, no que diz respeito à faixa etária, um estudo realizado na cidade de São Paulo, em 2014, mostrou que a maior prevalência da coinfecção por sífilis está em jovens (LUPPI et al., 2018), enquanto neste trabalho a faixa etária identificada foi acima de 60 anos.

É importante salientar, também, que existem consequências oriundas da coinfecção, que vão desde alterações na história e curso naturais da doença, repercutindo também na resposta ao tratamento. Uma das complicações está relacionada à progressão mais rápida para o sistema nervoso, causando a neurosífilis, resultado da baixa contagem de CD4. Dessa forma, destaca-se a importância da realização de testes rápidos de sífilis e HIV, na mesma ocasião, para diagnóstico e tratamento precoce (ARAÚJO et al., 2017).

\section{Conclusão}

Para os discentes o projeto de extensão Sexualidade Responsável representa uma oportunidade singular de aprendizagem direcionada para o desenvolvimento de habilidades práticas, de relações humanas, aprimoramento do conhecimento, aumento da produção científica e participação em eventos. Constitui, portanto, uma experiência que engrandece a formação profissional.

Nota-se que, além da detecção de IST, contribui-se socialmente no enfrentamento das IST com a disseminação de conhecimento durante as fases pré e pós teste, distribuição de métodos preventivos e, uma vez detectada a presença de alguma IST, facilitou-se a iniciação de tratamento e cuidados através de encaminhamento a outros locais da rede de atenção à saúde. Além disso, por meio do projeto, notou-se a necessidade de mais ações de educação em saúde sobre sexualidade nas unidades escolares. 
A realização do projeto expôs uma provável deficiência na formação dos alunos, caso não passassem por tais capacitações e envolvimento com a comunidade, notando-se uma significativa dificuldade inicial ao adquirir as habilidades técnicas para a realização de testes e desenvolver conversação de qualidade no pré e pós teste.

Como limitações, encontram-se as dificuldades para a sua realização em virtude da logística necessária para as capacitações e disponibilidade de testes rápidos e insumos. Porém, ressalta-se a importância de sua continuidade, uma vez que a extensão universitária gera bons impactos na formação dos alunos, ao mesmo tempo que contribui com a comunidade.

\section{Referências}

ANDRADE, H. S. et al. Caracterização epidemiológica dos casos de sífilis em mulheres. Ciência \& Saúde, Minas Gerais, v. 12, n. 1, jan./mar. 2019.

ANDRADE, J. et al. Vulnerabilidade de idosos a infecções sexualmente transmissíveis. Acta Paul Enferm., São Paulo, v. 30, n. 1, p. 8-15, 2017.

ARAÚJO, L. M. et al. Guia Prático em Abordagem Sindrômica: Prática Baseada em Evidências - Sífilis. 1. ed. Cuiabá: [s.n], 2017.

AZEVEDO, J. P. F. Diagnóstico tardio da infecção pelo HIV: Análise de fatores relacionados ao acesso aos serviços de saúde. 2017. Trabalho de Conclusão de Curso (Bacharelado em Enfermagem) - Universidade Federal de Campina Grande - PB, 2017.

BAGATINI, C. L. T. et al. Teste rápido para sífilis no pré-natal da atenção básica: avaliação institucional qualitativa e educação permanente em saúde. Saúde em Redes, v. 2, n. 1, p. 81-95, 2016.

BARBOSA, K. F. et al. Fatores associados ao não uso de preservativo e prevalência de HIV, hepatites virais B e C e sífilis: estudo transversal em comunidades rurais de Ouro Preto, Minas Gerais, entre 2014 e 2016. Epidemiol. Serv. Saúde, Brasília, v. 28, n. 2, p. 112, 2019.

BARROS, C. V. L. et al. Bio-behavioral survey of syphilis in homeless men in Central Brazil: a cross-sectional study. Cad. Saúde Pública, v. 34, n. 6, p. 1-13, out. 2018. doi: 10.1590/0102-311X00033317. Acesso em: 12 dez. 2020. 
BARTH, P.O.; BECK, S.T. Importância da implantação de Testes Rápidos para o diagnóstico de doenças com impacto na saúde pública: Revisão. Ciências da Saúde, Santa Maria, v. 19, n. 3, p. 537-548, 2018.

BOTTON, A.; CÚNICO, S. D.; STREY, M. N. Diferença de gênero no acesso aos serviços de saúde: problematizações necessárias. Revista Mudanças- Psicologia da Saúde, Rio Grande do Sul, v. 25, n. 1, p. 67-72, jan./jun. 2017.

BRASIL. Ministério da Saúde. Secretaria de Vigilância em Saúde. Departamento de DST, Aids e Hepatites Virais. A B C D E do Diagnóstico para as Hepatites Virais. 1. ed. Brasília: Ministério da Saúde, 2009.

BRASIL. Ministério da Saúde. Secretaria de Vigilância em Saúde. Departamento de DST, Aids e Hepatites Virais. HIV - Estratégias para utilização de testes rápidos no Brasil. Brasília: Ministério da Saúde, 2010.

BRASIL Ministério da Saúde. Secretaria de Vigilância em Saúde. Departamento de DST, Aids e Hepatites Virais. Diagnóstico de Hepatites Virais. Brasília: Ministério da Saúde, 2014.

BRASIL Ministério da Saúde. Secretaria de Vigilância em Saúde. Departamento de DST, Aids e Hepatites Virais. Protocolo Clínico e Diretrizes Terapêuticas (PCDT) - Atenção Integrada às Pessoas com Infecções Sexualmente Transmissíveis (IST). Brasília: Ministério da Saúde, 2015a.

BRASIL. Programa Conjunto das Nações Unidas sobre HIV/AIDS. 90-90-90: uma meta ambiciosa de tratamento para contribuir para o fim da epidemia de AIDS. Genebra, 2015b. Disponível em: http://unaids.org.br/wp-content/uploads/2015/11/2015 11 20 UNAIDS TRATAMENTO_META_PT_v4_GB.pdf. Acesso em: 12 dez. 2020.

BRASIL Ministério da Saúde. Secretaria de Vigilância em Saúde. Departamento de Vigilância, Prevenção e Controle das DST, Aids e Hepatites Virais. Manual Técnico para Diagnóstico da Sífilis. Brasília: Ministério da Saúde, 2016.

BRASIL Ministério da Saúde. Secretaria de Vigilância em Saúde. Departamento de Vigilância, Prevenção e Controle das IST, do HIV/Aids e das Hepatites Virais. Manual Técnico para o Diagnóstico das Hepatites Virais. Brasília: Ministério da Saúde, 2018a.

BRASIL Ministério da Saúde. Secretaria de Vigilância em Saúde. Departamento de Vigilância, Prevenção e Controle das DST, Aids e Hepatites Virais. Acolhimento, Aconselhamento e Orientações Pré e Pós-Teste. Brasília, 2018 b. 
BRASIL. Ministério da Saúde. Secretaria de Vigilância em Saúde. Coordenação-Geral de Desenvolvimento da Epidemiologia em Serviços. Guia de Vigilância em Saúde: volume único. 3. ed. Brasília: Ministério da Saúde, 2019a.

BRASIL Ministério da Saúde. Secretaria de vigilância em Saúde. Departamento de Doenças de Condições Crônicas e IST. Boletim epidemiológico - HIV/AIDS 2019. Brasília: Ministério da Saúde, dez. 2019b.

BRASIL Ministério da Saúde. Secretaria de Vigilância em Saúde. Coordenação-Geral de Desenvolvimento da Epidemiologia em Serviços. Protocolo Clínico e Diretrizes Terapêuticas para Prevenção da Transmissão Vertical do HIV, Sífilis e Hepatites Virais. Brasília: Ministério da Saúde, 2019c.

BRASIL Ministério da Saúde. Secretaria de vigilância em Saúde. Departamento de Doenças de Condições Crônicas e IST - DCCI. Boletim epidemiológico- Sífilis 2019. Brasília: Ministério da Saúde, out. 2019d.

BRASIL Ministério da Saúde. Secretaria de vigilância em Saúde. Departamento de Doenças de Condições Crônicas e IST - DCCI. Boletim epidemiológico- Hepatites Virais 2019. v. 50. Brasília: Ministério da Saúde, jul. 2019e.

BRASIL Ministério da Saúde. Secretaria de Vigilância em Saúde. Departamento de Doenças de Condições Crônicas e Infecções Sexualmente Transmissíveis. Protocolo Clínico e Diretrizes Terapêuticas para Atenção Integral às Pessoas com Infecções Sexualmente Transmissíveis (IST). Brasília: Ministério da Saúde, 2020.

BRUM, M. L. B.; MOTTA, M. G. C.; ZANATTA, E. A. Sistemas bioecológicos e elementos que vulnerabilizam adolescentes frente às infecções sexualmente transmissíveis. Texto Contexto Enferm, v. 28, p. 1-13, 2019.

CUNHA, L. C. Ampliando percepções sobre uso e acesso ao preservativo masculino por adolescentes e jovens: influências do Projeto Saúde e Prevenção nas Escolas. Dissertação (Mestrado em Saúde da Família) - Universidade Federal de Mato Grosso do Sul, Campo Grande, 2015.

FERREIRA, C. de O. et al. Vulnerabilidade a infecções sexualmente transmissíveis em idosos usuários de um centro de testagem e aconselhamento. Arq. Cienc. Saúde UNIPAR, Umuarama, v. 23, n. 3, p. 171-180, set./dez. 2019.

FONTE, V. R. F. et al. Conhecimento das infecções sexualmente transmissíveis entre universitários. Escola Anna Nery, v. 22, n. 2, 2018. 
FONTES, M. B. et al. Fatores determinantes de conhecimentos, atitudes e práticas em DST/Aids e hepatites virais, entre jovens de 18 a 29 anos, no Brasil. Ciência \& Saúde Coletiva, v. 22, n. 4, p. 1343-1352, 2017.

GOMES, E. S. S.; GALINGO, W. C. M. Equipes de Saúde da Família Frente à Testagem e ao Aconselhamento das IST, HIV-AIDS. Revista Baiana de Saúde Pública, Bahia, v. 41, n. 3, p. 628-649, 2017.

GRUMACH, A. S. et al. A (Des)Informação Relativa à Aplicação da Penicilina na Rede do Sistema de Saúde do Brasil: O Caso da Sífilis. DST - J bras Doenças Sex Transm, São Paulo, v. 19, n. 3-4, p. 120-127, 2007.

GUINSBURG, R.; SANTOS, A. M. N. dos. Critérios diagnósticos e tratamento da sífilis congênita. Documento Científico. Departamento de Neonatologia. Sociedade Brasileira de Pediatria, São Paulo, v. 20, dez. 2010.

LORETO, S.; AZEVEDO-PEREIRA, J. M. A infecção por HIV-Importância das fases iniciais e do diagnóstico precoce. Acta Farmacêutica Portuguesa, v. 11, p. 1-17, 2012.

LUPPI, C. G. et al. Fatores associados à coinfecção por HIV em casos de sífilis adquirida notificados em um Centro de Referência de Doenças Sexualmente Transmissíveis e Aids no município de São Paulo, 2014. Epidemiol. Serv. Saúde, v. 27, n.1, 2018.

MARTINS, A. M.; MODERNA, S. M.; Acesso da população masculina e utilização dos serviços de atenção primária à saúde em Belo Horizonte- MG. Revista atenção primária à saúde, Minas Gerais, v. 20, n. 4, p. 482-492, out./dez. 2017.

NUNES, G. M. S. et al. Triagem sorológica para HIV 1 e 2, sífilis, hepatites B e C, proveniente de ação de extensão universitária no município de Anápolis/go. In: MOSTRA CIENTÍFICA DE AÇÕES EXTENSIONISTAS, 10., v. 8. 2018, Anápolis. Livro de Resumos... Anápolis: UniEVANGÉLICA, 2018.

OLIVEIRA, T. O. et al. Perfil epidemiológico e características de coinfecções associadas às pessoas soropositivas. Rev enferm UFPE online, v. 13, p. 1-11, 2019.

PINTO, V. M. et al. Prevalência de sífilis na população em situação de rua. Rev Bras Epidemiol., São Paulo, p. 341-354, abr./jun. 2014. Disponível em https://www.scielo.br/pdf/rbepid/v17n2/pt 1415-790X-rbepid-17-02-00341.pdf. Acesso em: 12 dez. 2020. 
RUSSO, K.; ARREGUY, M. E.; Projeto "Saúde e Prevenção nas Escolas”: percepções de professores e alunos sobre a distribuição de preservativos masculinos no ambiente escolar. Revista de Saúde Coletiva, Rio de Janeiro, v. 25, n. 2, p. 501-523, 2015.

SANTOS, G. de S. Conhecendo a vulnerabilidade de HIV/AIDS de dois grupos de idosos. 2018. 76f. Dissertação (Mestrado em Enfermagem) - Centro de Ciências da Saúde, Universidade Federal da Paraíba, João Pessoa, 2018. Disponível em:

https://repositorio.ufpb.br/jspui/bitstream/123456789/12324/1/Arquivototal.pdf . Acesso em: 12 dez. 2020.

SILVA, A. M. S. et al. Fatores associados ao desenvolvimento da hepatite B. Caderno de Graduação - Ciências Biológicas e da Saúde UNIT, Alagoas, v. 5, n. 3, p. 39-48, nov. 2019.

SILVA, M. R. F. et al. Reflexões sobre as ações extensionistas e de pesquisa no combate à COVID-19 na universidade do estado do Rio Grande do Norte. Brazilian Journal of health Review., Curitiba, v. 3, n. 2, p. 3622-3646 mar./apr. 2020.

SILVA, P. A. S. et al. Sífilis em mulheres egressas do sistema prisional: prevalência e fatores associados. Rev Rene, v. 19, p. 1-8, 2018. DOI 10.15253/2175-6783.2018193321 Disponível em: http://periodicos.ufc.br/rene/article/view/32669/pdf. Acesso em: 12 dez. 2020.

SOUSA, C. J. O.; SOUSA, C. R. C. Resistência masculina pela atenção à saúde. Revista científica multidisciplinar núcleo do conhecimento, São Paulo, 9. ed., v.1, p. 5-12, dez. 2017. 